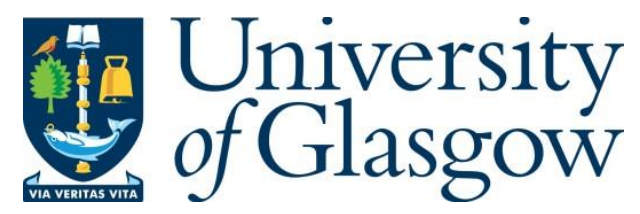

Qadir, J. and Imran, M. A. (2018) Learning 101: The untaught basics. IEEE Potentials, 37(3), pp. 33-38.

There may be differences between this version and the published version. You are advised to consult the publisher's version if you wish to cite from it.

http://eprints.gla.ac.uk/147253/

Deposited on: 4 September 2017

Enlighten - Research publications by members of the University of Glasgow

http://eprints.gla.ac.uk 


\title{
Learning 101: The Untaught Basics
}

\author{
Junaid Qadir and Muhammad Ali Imran
}

\begin{abstract}
Despite the accessibility of a wealth of information in the current era-in the form of books, universities, or online 'massive open online courses (MOOCs)-well-intentioned hardworking students often fail to learn effectively due to deficient learning technique or mindset. Two reasons in particular hinder students from achieving their potential: firstly, the intuition of students regarding how learning works is often flawed and counterproductive; and secondly, despite significant progress in the research discipline of "learning sciences", these hard-earned scientific insights have not yet filtered their way through the research community onto the students who stand to benefit most from this knowledge. In this article-titled "Learning 101: the untaught basics"-we aim to popularize the important insights learnt by the learning science researchers by making it accessible to students, who continue to largely use suboptimal intuitive learning techniques not knowing any better. The benefits of knowing these insights are immediate and substantial: we can use this knowledge to understand and calibrate our learning; it can also facilitate efficient learning (learning more in less time and avoiding "labor in vain").
\end{abstract}

\section{COMMON LEARNING IMPEDIMENTS}

To learn optimally, we need to not only discover how to learn effectively, but also to identify and avoid common learning impediments. In our previous work [7], we identified seven common learning impediments (shown in Table I). These learning impediments emerge from deficient mindset $(1,4)$, focus $(2,3)$, support $(5,6)$, and metacognition (7). These learning impediments are not insurmountable: three potential remedies for each learning impediment are identified in [7] as shown in Table I. A major impediment-arguably the mother impediment of all the listed impediments, if considered broadly - is "Not Learning How to Learn" (7). The overarching remedy for this impediment is to spend time to develop better metacognitive skills. Our aim in this article is to succinctly present the relevant research findings in an accessible manner so that learners can use this knowledge to improve their metacognitive skills and learning strategies.

\section{LEARNING HOW TO LEARN}

"The most useful piece of learning for the uses of life is to unlearn what is untrue"-Antisthenes

Despite our intimacy with the task of learning (through the trials and errors of everyday living), research has shown that our metacognitive judgment about learning-i.e., our intuitive understanding of how we learn-is flawed [2] and many of our well-established and commonly-accepted intuitive notions are not only incognizant of scientific findings about learning but oppose the optimal course of action. Unfortunately, the common societal attitude, and the general assumption in colleges
TABLE I: Common Learning Impediments \& Remedies (Reproduced From Qadir [7])

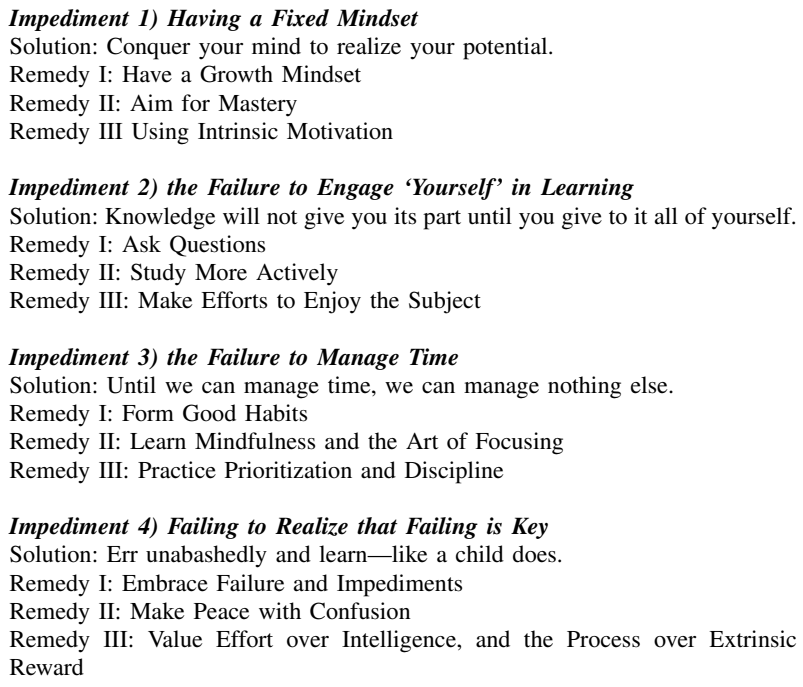

For a more detailed elaboration of these impediments, see [7].

and universities, is that learners can automatically figure out the art and science of learning themselves. This is reflected in the way we emphasize the teaching of content and subjects in learning institutions but not the dissemination of information regarding optimal learning strategy.

There is a Taoist saying that in order to gain knowledge, one should add something every day, but to gain wisdom, one should subtract something every day. We propose that learners should supplant the erroneous intuitive learning model with an improved metacognitive model of how learning actually works. This exercise is especially timely keeping in mind 
the abundant learning opportunities in the modern worlde.g., in the form of free open educational resources (OER), such as massive open online courses (MOOCs) - that present unprecedented opportunities for lifelong learning. We feel that these abundant resources will only lead to learning advances when the students are equipped with effective learning skillsskills they can acquire by mastering the contents of the oftuntaught "Learning 101" course, the subject that we aim to elaborate through this article.

\section{TO LEARN, WE MUST FIRST UNLEARN: WHY LEARNING CAN BE PARADOXICAL?}

"True wisdom comes to each of us when we realize how little we understand about life, ourselves, and the world around us."-Socrates.

Our intuition about learning is usually centered on a shortterm vision of fluency, which may not translate and generalize to other settings and which is transient and fleeting. The "paradoxes of learning" arise due to the apparent dissonance between how learning actually works (particularly in the long term) and how we think it works. In many cases, our minds are fixated over some ideals that are not desirable from the point of view of learning: we call such fixations "undesirable fixations". On the other hand, many seemingly undesirable difficulties can have desirable consequences: we call such difficulties "desirable difficulties". To become sophisticated learners, we must lose our undesirable fixations and instead embrace desirable difficulties.

\section{DESIRABLE DIFFICULTIES}

While we intuitively dislike difficulties and thus try to avoid them, many difficulties (but not all) have a positive effect on learning. The well-known cognitive psychologist Bob Bjork coined the term "desirable difficulties" [2] for such difficulties that have a positive effect on learning.

\section{1) Effortful learning is better learning}

"Practice that's spaced out, interleaved with other learning, and varied produces better mastery, longer retention, and more versatility. But these benefits come at a price: when practice is spaced, interleaved, and varied, it requires more effort."Brown, Roediger, and McDaniel [3]

- A famous aphorism goes as "No Pain No Gain". While we admire effortless learning, research shows that effortless learning is mostly transitory. Learning-i.e., actual learning that requires the ability to remember and transfer concepts in the long term-requires effort and reaching out. Independent and active learning also appears to be difficult but it also leads to better longlasting learning. Unfortunately, many learners back off at the slightest hint of discomfort or prematurely conclude their lack of feel for the subject.
- Most effective learning techniques are effortful: Research literature in the learning sciences has documented the efficacy of a number of effective learning techniques-e.g., retrieval practice, and the use of spacing and interleaving-most of which are effortful. Retrieval practice - recalling facts or concepts or events from memory-is a particularly effective learning strategy. One of the most striking research findings of the learning sciences is the efficiency of testing and retrieval practice - and the more effortful the retrieval, the stronger the benefit [3]. Another powerful but effortful technique is spaced learning (to space out the learning process and to avoid cramming material without practicing it subsequently) and interleaving (not studying something in isolation but mixing the subject and task being studied with other things). Research has shown that while retrieval is harder with spaced learning and interleaving, resulting in the feeling that the learning is less accomplished, the resulting learning is actually deeper and will lead to easier retrieval in the future.

\section{2) To Learn, You Must Forget, Then Interrupt Forgetting}

"It is only what breaks that grows."-Unknown.

- The futility of cramming: While cramming can result in short-term learning benefits, which is why it can even lead to good results for students who study just before an exam, the knowledge gained is fleeting and drifts away as soon as it was gained. Learning is a natural system that evolves at a natural rate-just like agricultural systems have their natural cycles of harvest, and you cannot cram your sowing and expect an amplified harvestlearning also happens naturally in a slow and steady fashion. Students often assume that massed reading and overlearning in one sitting is a good learning technique due to the resulting fluency. This "fluency illusion"and not anxiety or poor luck or unfairness or uneven stupidity - is most often the culpable culprit behind poor learning and unexpectedly poor test performances [4]. Just like a body builder knows not to get fooled by the temporary muscle pump after an intensive exercise, a student would do well not to get duped by the evanescent feeling of fluency.

- Using forgetting and learning symbiotically: It is often thought that the central challenge to improving the way we learn is to eliminate or overpower the process of forgetting. It is true that periodic practice is necessary since it arrests forgetting and strengthens the retrieval routes. But while we stigmatize forgetting, we must remember that forgetting is a necessary corollary to learning, and forgetting and learning work together symbiotically and together form the part and parcel of learning [1]. Recent research is establishing a friendly, rather than an adversarial, role of forgetting. In particular, practice before forgetting is wasteful, while practice after forgetting leads to longer-lasting learning. 


\section{3) Disfluency/Uncomfort Can Be Good for Learning}

"That which does not kill us makes us stronger"-Nietzsche

- The benefits of slowing down learning: In the modern world of abundant open high-quality resources such as MOOCs, the combination of the propensity for immediate gratification, the loss of the ability to focus, and the illusive power of fluency masqureading as learning has become toxic for deep learning and it will help here to recognize that slower can be better especially for long-term learning. Many celebrated "geniuses" (e.g., Einstein, Edison, Darwin) were actually considered slow learners in school. It has been shown in cognitive research that slowing down learning or making it hardere.g., by omitting letters from words in a text-can actually improve retention of material. This is because this forces the student to be more active (e.g., by working harder in supplying the missing letters). In the pithy formulation of Daniel Willingham, memory is the residue of thought. Active learning can also be engaged profitably by asking a student to solve a problem before being taught, even if errors are made in the attempt (as long as the correct solution is thereafter provided); this happens because the student's attempt to answer the question activates a search for relevant prior information and patterns in a way that helps the student retain the knowledge much more than simply reading would have [3].

- The upside of confusion \& perplexity: John Dewey is known to have noted that the origin of thinking is some perplexity, confusion, or doubt-since we reflect when such event arises and not when everything goes smoothly. Although we dislike effortful grappling with confusions, these activities serve as the stepping stone towards mastery. Becoming smart entails the feeling of being dumb during learning.

\section{UNDESIRABLE FIXATIONS}

Due to our flawed intuitive model of how learning works, most learners are unduly, and counter-productively, fixated on perfection, fluency, and discipline as learning ideals. We discuss next why these fixations are undesirable.

\section{1) Fixating on perfection: Why mistakes are required?}

"An expert is a man who has made all the mistakes which can be made, in a narrow field"-Niels Bohr

- Understand the importance of errors and mistakes: In general, learners view errors and mistakes as something to avoid in toto (since mistakes are often interpreted by oneself and by others as the learner's inadequacy). Mistakes however serve as the necessary stepping stones to mastery and advanced learning. Any learning activity that aims to eliminate all errors can lead to superficial and fragile learning. By becoming open to mistakes, learners will be more amenable to embrace learning practices laced with desirable difficulties that lead to better long-lasting learning outcomes.

- The mirage and futility of chasing perfection: Learning without mistakes is fragile and dangerous, since such learning is typically the result of fluency rather than mastery. It's important to allow for mistakes to happen through extensive exploration of the subject through other viewpoints, spaced learning, interleaving, etc. Fixating on perfection also engenders a psychological mindset—called a fixed-mindset [5] - that is not conducive to learning. Learners with fixed mindset see events and circumstances as a direct measure of competence and worth. Students with fixed mindsets obsess over documenting their intelligence, instead of focusing on developing their intelligence.

\section{2) Fixating on fluency: Why fluency is not sufficient?}

"Rising familiarity with a text and fluency in reading it can create an illusion of mastery."-Brown, Roediger, and McDaniel [3].

- The Fluency Illusion: The fluency illusion underlies the widespread adoption of suboptimal learning techniques such as rereading and massed practice that engender fluency but are inefficient compared to other learning techniques [3]. The fluency illusion is arguably the biggest cause of our flawed learning intuitions. One reason for its significant role in messing up our intuition is that fluency feels good. To further complicate matters, techniques conducive to long-term learning (such as spaced learning, retrieval practice, interleaving) initially manifest disfluency. Such techniques therefore do not appeal to learners since the learning appears slower and devoid of the rapid improvements that massed practice can demonstrate. But fluency-when not strengthened by repetition and retrieval exercises - is deceptively transient. Although commonly conflated, it is important for all learners to remember that fluency and mastery are two very different things.

- Massed practice (and rereading) is suboptimal: The strategy of rereading text and massed practice (contiguous practice that is not spaced out) is the technique of choice for many learners since it aligns well with the intuition of learners honed on a mindset that emphasized learning in the short term and equates fluency with learning. As noted by Brown et al. [3], rereading as a learning technique suffers from three problems: (i) it is more consuming; (ii) it does not result in durable memory since the forgetting process has not yet set in; and (iii) it involves unwittingly a deceptive feeling of faux mastery that comes with the growing familiarity with the text.

\section{3) Fixating on discipline: The upside of variety}

"You don't understand anything until you learn it more than one way."-Marvin Minsky 
- Idolization of focus and the Einstellung effect: Discipline and focus are celebrated-not without reasonas virtuous facilitators of success. But too much of a focus on discipline can prove itself to be an overkill for the purpose of learning, and a pluralistic and varied approach that allows for some serendipity may be better suited. For example, while we do need to focus intently for problem solving, too much of focus can block us from accessing fresh ideas. The more focused we're on producing creative solutions quickly, the least likely we are to be successful. This phenomenon is called Einstellung and has been empirically demonstrated in numerous experiments [6]. Focusing intensely on a creative task in a single setting, especially when one is stuck may be the worst possible strategy; sometimes all you need is a break to freshen up your thinking. Even though we stigmatize procrastinating, but procrastinating may be good in certain learning situations especially when we want to arrive at creative solutions.

- Variety is the spice of learning: Variety of environmental stimuli and input modality is also very important for learning. There is no formula for the complex process of learning, but if we had to come up with simple one, "Mastery = Understanding + Repetition (In Varied Settings)" would be a good candidate. The learning potency of variety is also demonstrated in the empirical efficacy of interleaving (i.e., interspersing various topics together) and the positive interleaving effect can be explained on the basis that it allows for encoding and embedding of new knowledge in existing networks of prior perceptions, facts, and thoughts.

\section{FOR EFFICIENT LEARNING, USE OPTIMAL LEARNING TECHNIQUES}

Rereading text and massed practice of a skill or new knowledge are by far the preferred study strategies of learners of all stripes, but they're also among the least productive."Brown, Roediger, and McDaniel [3]

\section{A. Testing/ Retrieval Practice as a Learning Tool}

"In virtually all areas of learning, you build better mastery when you use testing as a tool to identify and bring up your areas of weakness." —-Brown, Roediger, and McDaniel [3].

Despite getting a bad rap, testing-or retrieval practice, as it is customarily called in learning sciences-is a very powerful but underestimated learning technique. Retrieval practice or testing methods include, inter alia, quizzes and exams in formal assessment settings; self-test (e.g., through flashcards); and oral examination. In our context, testing simply implies the action of forcing learners to recall the learned information from memory. It has been shown that a single simple quiz can help produce better learning compared to techniques that are most popular with students (rereading of a text, and reviewing of notes) [3]. The potency of testing or retrieving information from human memory arises from the fact that retrieval is a "memory modifier" [2] rather than simply some inert playback -in particular, retrieved information, rather than being left in the same state, becomes more recallable in the future. Testing can help learners achieve three crucial aims: (1) by supporting recall, the retention of knowledge is enhanced; (2) by enhancing self-awareness, learners become more aware of their mental processes and can better understand what they do and do not know; (3) by putting learners in controlled adversity, important non-cognitive skills such as resilience and grit can be developed.

\section{B. Spaced Learning and Interleaving}

"The truth is, nothing in learning science comes close [to spaced learning] in terms of immediate, significant, and reliable improvements to learning."-Benedict Carey [4].

There is a substantial body of research that has demonstrated the power of spaced learning. Although brain is not like a muscle in any straightforward sense and is much more eccentric, it is instructive to note that massed learning can give temporary fluency, just like a body builder can pump muscles temporarily by cramming exercises. However, growth only occurs with a spaced exercise routine (in which exercise and rest follow each other cyclically). Similarly, long-term learning also requires spaced practice and does not result from cramming. There are two main benefits to spaced learning: firstly, it requires the exercise of retrieving from long-term memory; secondly, the time between the spaced learning sessions allows our minds to better organize and interconnect the new knowledge with prior knowledge.

The related technique of interleaving encourages the incorporation of variety by suggesting that practice of different knowledge or skills should be performed together. Such a practice allows for better recognition of the context of the knowledge or skill being learnt. This allows for better discrimination and allows for better problem solving skills as the learners begins to get a grip on what techniques to use in what situations. Although retrieval will feel harder with interleaving, the effort produces longer lasting learning and a more versatile ability to generalize and apply the knowledge correctly as per the context.

\section{Aiming for Mastery}

"To be a sophisticated learner requires understanding that creating durable and flexible access to to-be-learned information is partly a matter of achieving a meaningful encoding of that information and partly a matter of exercising the retrieval process."-Bjork, Dunlosky, and Kornell.

Becoming an effective learner is much more than becoming fluent. For optimal learning-i.e., learning with efficiency (most learning for a given time), flexibility (the ability to generalize to new situations), and longevity (the ability to remember the knowledge for a long time)-one must understand a subject, encode it in storage memory elaborately amd memorably, and then practise it in a variety of settings. As 
noted by Bjork et al. [2], becoming a sophisticated learner entails partially a meaningful encoding of learnt information (into the storage memory) and partially the exercising of the retrieval process (through retrieval memory). This requires familiarization with activities and techniques that can enhance storage and subsequent retrieval of the content and procedure to be learnt. In addition, one must also become aware of certain illusions and biases that can impede learning.

Previous research has demonstrated that one important difference between the knowledge of experts and novices is that the former have much more dense connections between the various concepts, facts, and skills that they have [4]. The strength of encoding in memory (to be specific, the storage memory) depends on how connected the knowledge being learnt is to other previously acquired knowledge. An attempt to connect new knowledge with prior knowledge will pay dividends as the new knowledge become well entrenched. In this regard, researchers have shown the power of the unconscious and the role of sleep and rest in learning. In the words of the cognitive psychologist Richard Nisbett, one should never fail to take advantage of the free labor of the unconscious mind.

\section{SUMMARY}

To learn, you must work with zest, you must invest effort into learning, recognize and resist the illusions of learning (such as confusing fluency with mastery). To benefit from optimal learning techniques shown in literature, the following recommendations are provided:

1) Spaced out learning: You should space out, rather than concentrate, your learning sessions.

2) Interleaving: You should interleave, rather than block, study/practice sessions on separate to-be-learned topics.

3) Testing: You should test yourself on the learnt knowledge and resist the urge of looking things up and rereading.

4) Variety: You should vary the conditions of your learning - even the environmental context of studyingand avoid learning always in the same modality and environment.

Although, the techniques above are efficient in terms of long-term learning, the caveat is that using these techniques may result in more effortful even uncomfortable experiences. How then to ensure that these efficient but effortful techniques are adopted? One way is to develop a growth mindset, through which you recognize that intelligence is not a fixed trait but is something that can grow with effort, dedication, and hard work. Mistakes, forgetting, effort must not be construed as a sign of lack of learning competence but as a necessary stepping stone to substantial learning.

Armed with these new Learning 101 insights, it is hoped that learners will adopt more efficient learning techniques in preference to suboptimal techniques and thereby become better independent, self-regulated, and sophisticated learners.

\section{About the authors}

Junaid Qadir (junaid.qadir@itu.edu.pk) is an Associate Professor at the Information Technology University (ITU) Punjab, Lahore since December 2015. He is currently serving as the director of the IHSAN research lab at ITU. He is broadly interested in matters related to learning, teaching, and education. He was awarded the Higher Education Commission (HEC)'s (Pakistan) Best University Teacher Award for the year 2012-2013.

Muhammad Ali Imran (muhammad.imran@glasgow.ac.uk) is a Professor in Communication Systems in University of Glasgow, Vice Dean of Glasgow College UESTC and Program Director of Electrical and Electronics with Communications. $\mathrm{He}$ has been awarded the FEPS Learning and Teaching award 2014 and twice nominated for Tony Jean's Inspirational Teaching award. He is a Senior Fellow of Higher Education Academy (SFHEA), UK.

\section{REFERENCES}

[1] Robert A Bjork and AS Benjamin. On the symbiosis of remembering, forgetting, and learning. Successful remembering and successful forgetting: A Festschrift in honor of Robert A. Bjork, pages 1-22, 2011.

[2] Robert A Bjork, John Dunlosky, and Nate Kornell. Self-regulated learning: Beliefs, techniques, and illusions. Annual review of psychology, 64:417-444, 2013.

[3] Peter C Brown, Henry L Roediger, and Mark A McDaniel. Make it stick. Harvard University Press, 2014.

[4] Benedict Carey. How we learn. Macmillan, 2014.

[5] Carol Dweck. Mindset: The new psychology of success. Random House LLC, 2006.

[6] Barbara Oakley. A mind for numbers: how to excel at math and science (even if you flunked algebra). Penguin, 2014.

[7] Junaid Qadir. What every student should know: Seven learning impediments and their remedies. Potentials, IEEE, 34(3):30-35, 2015. 